\title{
Clinical field note - Ultrasound Therapy: Getting it Right!
}

\author{
Amusat, $\mathbf{N}$. \\ Stroke and Geriatric Empowerment Program, Two Hills Health Centre, Two Hills, Alberta, Canada \\ Email:sepet69@hotmail.com

\section{SUMMARY} \\ Ultrasound therapy is a popular physical therapy modality among clinicians. The era of evidence-based practice \\ has, however, led to intense research evaluation of its effectiveness. In many systematic reviews and meta- \\ analyses on ultrasound therapy, multiple factors have resulted in inconclusive results on its possible \\ effectiveness. Recent information highlights the need to have adequate insonation energy for the desired effect \\ from the use of ultrasound therapy. Incorporating this vital information has led to a turn around in the evidence \\ of ultrasound research and, ultimately to the clinical use of this modality.
}

KEY WORDS: ultrasound, treatment, parameters, effectiveness

\section{INTRODUCTION}

Ultrasound (US) therapy is a widely-used treatment in physical therapy (PT) (Lindsay, Dearness and McGinley, 1995; ter Haar, Dyson and Oakley, 1985; Wong, Schumann, Townsend and Phelps, 2007). The popularity of this modality extends even to specialist orthopaedic physical therapists (Wong et al., 2007). Despite its wide application in clinical practice, there has not been enough research evidence to support its widespread use (Gam and Johannsen, 1995; Green, Buchbinder and Hetrick, 2003; Philadelphia Panel, 2001; van der Windt et al., 1999). Why then has US therapy continued to be popular and to be used among clinicians? The answer may not be simple. It has been suggested that anecdotal evidence of the effectiveness of US therapy has sustained its use among clinicians (Amusat, 2007). Also, some authors have challenged the conclusion that therapeutic ultrasound was ineffective because of the weak evidence available (Brockow, Franke, \& Resch, 1998). There are clear indications of a disconnection between the views held by the research and clinical communities (a significant use by clinicians while researchers could not find convincing evidence of its effectiveness). Ethically, a state of equipoise (or uncertainty) probably exists on the effectiveness of US therapy (Amusat, 2007; Freedman, 1987). Analysis of earlier systematic reviews and meta-analyses indicated that some of the previous studies had methodological deficiencies, and recommendations for more quality studies were made (Gam, and Johannsen, 1995; Green, Buchbinder and Hetrick, 2003; van der Windt et al., 1999).
A recent account indicated that no published review for the effects of US therapy on the shoulder has included newer studies (published since 1999) (Alexander et al., 2010). Enough may therefore not have been done (at least since 1999) to further disturb the state of uncertainty about the effectiveness of US therapy.

Recently, progress was reported in the science of US therapy. Studies using significantly less insonation energy reported that there was no positive effect of US therapy (Alexander et al., 2010). These authors reviewed 8 randomized trials that evaluated the effect of US therapy on soft tissue disorders of the shoulder. The minimum energy level determined to be of benefit was 2250 Joules/session (Alexander et al., 2010). Low energy exposure and very wide variability in the treatment parameters during insonation were some of the reasons for the inadequacy of US therapy studies. Studies utilizing low doses of US therapy are in fact only performing pseudo ultrasound treatment, that may not be expected to be effective (Brockow, Franke, and Resch, 1998). It then becomes imperative, not only in US therapy studies, but in physical therapy to strive for a standardization of treatment protocols to improve the internal validity of PT effectiveness studies (Amusat, 2005).

The aim of this clinical field note was to provide clinical examples of calculating appropriate US therapy energy, while applying the information from the study of Alexander and colleagues (2010). This is to make the information widely available to PTs who have had no access to the original publication. 


\section{Calculation of appropriate dose of US energy (Houghton, 2009)}

Spatial average temporal average (SATA)

Spatial average peak intensity (SATP, W/ $\mathrm{cm}^{2}$ )

Spatial average temporal average $($ SATA) $=$ Spatial average peak intensity (SATP, $\mathrm{W} / \mathrm{cm}^{2}$ )

Intensity $=$ SATA $\left(\mathrm{W} / \mathrm{cm}^{2}\right) \times$ duty cycle $(\%)$

Total energy per session (Joules) = Intensity $\left(\mathbf{W} / \mathbf{c m}^{2}\right)$

$x$ time (seconds) $x$ US head size $\left(\mathrm{cm}^{2}\right)$

Total energy exposure (Joules) $=$ Total energy $/$ session $x$ number of treatments.

Note: Recommended energy $>2250$ Joules/session (SATA of $0.5-2 \mathrm{~W} / \mathrm{cm}^{2}$, average exposure times over 4 weeks of 5 hours) (Houghton, 2009)

\section{EXAMPLES}

Note that the parameters below were chosen to show how to calculate the appropriate energy of insonation in each situation. The main preoccupation should be to arrive at an energy level greater than 2250 Joules per treatment with combinations of US intensity, time of insonation and ultrasound head size. Most US therapy machines come with head sizes of 2,5 and $10 \mathrm{~cm}^{2}$. The choice of US therapy head size depends on the extent of the area under treatment. Apart from treating toes and fingers, most therapists use the $5 \mathrm{~cm}^{2}$ head. Therefore, I decided to use the $5 \mathrm{~cm}^{2}$ head in the calculation below. The intensity, time of insonation and US treatment head can be varied to suit the particular clinical situation, but still with the goal of achieving at least 2250 Joules of US energy per treatment session.

\section{1.}

Calculate the energy/session used to treat a chronic

left MCL sprain with the parameters below.

Parameters: $1 \mathrm{~W} / \mathrm{cm}^{2}, 100 \%$ duty cycle (continuous), 8 minutes, with a $5 \mathrm{~cm}^{2}$ ultrasound head

Intensity $=$ SATA $\left(\mathrm{W} / \mathrm{cm}^{2}\right) \mathrm{x}$ duty cycle $(\%)=1 \mathrm{~W} / \mathrm{cm}^{2} \mathrm{x}$ $1.0(100 \%$ duty cycle $)=1 \mathrm{~W} / \mathrm{cm}^{2}$

Time $=8 \times 60=480$ seconds

Total energy $($ Joules $)=$ Intensity $\left(\mathrm{W} / \mathrm{cm}^{2}\right) \times$ time $($ seconds $)$ $x$ US head size $\left(\mathrm{cm}^{2}\right)$

Energy $=1 \mathrm{~W} / \mathrm{cm}^{2} \times 480$ seconds $\times 5 \mathrm{~cm}^{2}=2400$ Joules
2. Calculate the energy/session used for an acute left ankle sprain with the parameters shown below.

Parameters: $1 \mathrm{~W} / \mathrm{cm}^{2}, 50 \%$ duty cycle (pulsed), 15 minutes, with a $5 \mathrm{~cm}^{2}$ ultrasound head

Intensity $=$ SATA $\left(\mathrm{W} / \mathrm{cm}^{2}\right) \mathrm{x}$ duty cycle $(\%)=1 \mathrm{~W} / \mathrm{cm}^{2} \mathrm{x}$ $0.5(50 \%$ duty cycle $)=0.5 \mathrm{~W} / \mathrm{cm}^{2}$

Time $=15$ × $60=900$ seconds

Total energy $($ Joules $)=$ Intensity $\left(\mathrm{W} / \mathrm{cm}^{2}\right) \times$ time $($ seconds $)$ $x$ US head size $\left(\mathrm{cm}^{2}\right)$

Energy $=0.5 \mathrm{~W} / \mathrm{cm}^{2} \times 900$ seconds $\times 5 \mathrm{~cm}^{2}=\mathbf{2 2 5 0}$ Joules.

3. Calculate the US energy/session used for an acute left lateral elbow sprain with the parameters below.

Parameters: $0.5 \mathrm{~W} / \mathrm{cm}^{2}, 50 \%$ duty cycle (pulsed), 10 minutes, with a $5 \mathrm{~cm}^{2}$ ultrasound head

Intensity $=$ SATA $\left(\mathrm{W} / \mathrm{cm}^{2}\right) \times$ duty cycle $(\%)=0.5 \mathrm{~W} / \mathrm{cm}^{2}$ x $0.5(50 \%$ duty cycle $)=0.25 \mathrm{~W} / \mathrm{cm}^{2}$

Time $=10 \times 60=600$ seconds

Total energy $($ Joules $)=$ Intensity $\left(\mathrm{W} / \mathrm{cm}^{2}\right) \times$ time $($ seconds $)$ $x$ US head size $\left(\mathrm{cm}^{2}\right)$

Energy $=0.25 \mathrm{~W} / \mathrm{cm}^{2} \times 600$ seconds $\mathrm{x} 5 \mathrm{~cm}^{2}=750$ Joules.

\section{COMMENTS}

The choice of $3 \mathrm{MHz}$ or $1 \mathrm{MHz}$ will depend on the depth of the tissue being treated and is not factored into the calculation of the total energy needed for treatment. As seen above, for an ultrasound head size of $5 \mathrm{~cm}^{2}$, the clinician has to adjust either the SATA $\left(\mathrm{W} / \mathrm{cm}^{2}\right)$ or the time to achieve the necessary energy of insonation. For the pulsed mode (duty cycles less than $100 \%$ ), more time will be needed per treatment. The energy of insonation was adequate for examples 1 and 2, but not adequate for example 3 .

\section{CONCLUSION}

Based on the recently available information, we now know that adequate US therapy parameters (giving optimal US energy) can deliver therapy benefits. This field note has provided clinicians with examples that could guide in the calculations of US therapy energy for treatments.

\section{Acknowledgements}

The author would like to thank Bello Ajediran (PT) and Lukman Ganiyu (PT) for the review of the manuscript and their useful comments. 


\section{References}

Amusat, N.T. 2007. On a Survey of Therapeutic Ultrasound. ... Wong et al. Physical Therapy 2007: 87:986-994 [letter]. Physical Therapy 87, 1558-1559, author reply 1559.

Amusat, N.T. 2005/ Quality of interventions: Establishing a benchmark [rapid response], to Robert, D. and Kari, B. 2005: Analysis of quality of interventions in systematic reviews. $B M J 331,507-509$.

Alexander, L.D., D.R.D. Gilman, D.R.. Brown, J.L. Brown and P.E. Houghton. 2010. Exposure to low amounts of ultrasound energy does not improve soft tissue shoulder pathology: A systematic review. Physical Therapy 90: 14-25.

Brockow, T., A. Franke and K.L. Resch. 1998. Physiotherapy for soft tissue shoulder disorders. Conclusion that therapeutic ultrasound is ineffective was based on weak evidence. $B M J$ 316, 555, author reply 556.

Freedman, B. 1987. Equipoise and the Ethics of Clinical Research. New England Journal of Medicine 317: 141-145.

Gam, A.N., F. Johannsen. 1995. Ultrasound therapy in musculoskeletal disorders: A meta-analysis. Pain 63, 85-91.

Green, S., R. Buchbinder and S. Hetrick. 2003. Physiotherapy interventions for shoulder pain. Cochrane Database Systematic Reviews 2003(2):CD004258.
Houghton, P. 2009. Ultrasound treatments involving $0.5 \mathrm{~W} / \mathrm{cm}^{2}$, $20 \%$ duty cycle, for 5 minutes are not enough. Canadian Physiotherapy Association Orthopaedic Division Review, Sept/Oct 2009, 35-36.

Lindsay, D.M., J. Dearness and C.C. McGinley. 1995. Electrotherapy usage trends in private physiotherapy practice in Alberta. Physiotherapy Canada 47: 30-34.

Philadelphia Panel. 2001. Philadelphia panel evidence-based clinical practice guidelines on selected rehabilitation interventions for shoulder pain. Physical Therapy 81: 1719-1730.

Ter Haar, G., M, Dyson and E.M. Oakley. 1985. The use of ultrasound by physiotherapists in Britain. Ultrasound Med Biol 13: 659-663.

Van der Windt, D.A., G.J. van der Heijden and S.G. van den Berg et al. 1999. Ultrasound therapy for musculoskeletal disorders: A systematic review. Pain 81: 257-271.

Wong, R.A., B. Schumann, R. Townsend and C.A. Phelps. 2007. A survey of therapeutic ultrasound use by physical therapists who are orthopaedic certified specialists. Physical Therapy 87: 986-994, discussion 995-1001. 